\title{
A NEW APPROACH FOR GENERALISED UNSHARP MASKING ALOGORITHM
}

\author{
Sunkari Sridhar ${ }^{1}$, Shaik Meeravali ${ }^{2}$ \\ ${ }^{1}$ Assistant Professor, ${ }^{2}$ Professor\& HOD, Department of ECE, RRSCET, Muthangi, Andhra Pradesh, India \\ sridharsunkari@gmail.com, shaik_5045@gmail.com
}

\begin{abstract}
We propose a new generalized algorithm using the exploratory data model as unified frame work. Enhancement of contrast and sharpness of an image is required in many applications. In applications like medical radiography enhancing movie features and observing the planets it is necessary to enhance the contrast and sharpness of an image. Unsharp masking is good tool for sharpness enhancement; it is an anti blurring filter. By using unsharp masking algorithm for sharpness enhancement, the resultant image suffering with two problems, first one is a hallo is appear around the edges of an image, and second one is rescaling process is needed for the resultant image. The aim of this paper is to enhance the contrast and sharpness of an image simultaneously and to solve the problems. In the proposed algorithm, we can adjust the two parameters controlling the contrast and sharpness to produce the desired output. The proposed algorithm is designed to address issues:1) simultaneously enhancing contrast and sharpness by means of individual treatment of the model component and the residual,2)reducing the halo effect by means of an edge-preserving filter using Bilateral filter. Experimental results, which comparable to recent published results, shows that proposed algorithm is able to significantly improve the sharpness and contrast of an image. This makes the proposed algorithm practically useful.
\end{abstract}

Index Terms: Bilateral filter, edge-preserving filter, exploratory data model, Image Enhancement, Unsharp Masking $* * *$

\section{INTRODUCTION}

Digital image processing allows the use of much more complex algorithms for image processing, and hence can offer more sophisticated performance at simple tasks.

An image is defined as a two dimensional light intensity function ( $\square$,), where $\square$ and $\square$ are spatial coordinates, and the value $f$ at any pair of coordinates $(\square$,$) is called intensity or$ grey level value of the image at that point. We require simultaneous enhancement of sharpness and contrast in many applications. Based on this requirement a continuous research is going on to develop new algorithms. We are having deferent type sharpness enhancement techniques, among these unsharp masking will gives enhanced sharpness with original image as background. We find some unwanted details in the resultant image. To avoid these we used new algorithms.

In this section firstly we discuss related works, which are sharpness enhancement techniques including unsharp masking, contrast enhancement and generalized linear system.

\subsection{Sharpness Enhancement}

\subsubsection{High pass filter}

The principal objective of sharpening is to highlight fine detail in an image or to enhance detail that has been blurred, either in error or as a natural effect of a particular method of image acquisition. Uses of image sharpening vary and include applications ranging from electronic printing and medical imaging to industrial inspection and autonomous guidance in military systems.

\begin{tabular}{|c|c|c|}
\hline$-1 / 9$ & $-1 / 9$ & $-1 / 9$ \\
\hline$-1 / 9$ & $8 / 9$ & $-1 / 9$ \\
\hline$-1 / 9$ & $-1 / 9$ & $-1 / 9$ \\
\hline
\end{tabular}

(a) High pass filter mask

\begin{tabular}{|c|c|c|}
\hline $\mathrm{Z}_{1}$ & $\mathrm{Z}_{1}$ & $\mathrm{Z}_{1}$ \\
\hline $\mathrm{Z}_{1}$ & $\mathrm{Z}_{1}$ & $\mathrm{Z}_{1}$ \\
\hline $\mathrm{Z}_{1}$ & $\mathrm{Z}_{1}$ & $\mathrm{Z}_{1}$ \\
\hline
\end{tabular}

(b) Image gray level values
Fig-1: $3 \times 3$ High pass Filter mask and image gray level values.

That is, with reference to the response of the mask at any point in the image is given by

\section{FLOWCHART}

$$
\begin{gathered}
R=1 / 9 *(-\mathrm{Z} 1-\mathrm{Z} 2-\mathrm{Z} 3-\mathrm{Z} 4+(8 * \mathrm{Z} 5)-\mathrm{Z} 6-\mathrm{Z} 7-\mathrm{Z} 8-\mathrm{Z} 9) \\
=1 / 9 *(-\mathrm{Zi} 9 \mathrm{i}=0+(2 * \mathrm{Z} 5))
\end{gathered}
$$

Where $\mathrm{Zi}$ is the gray level of the mask of the pixel associated with mask coefficient 1 . As usual, the response of the mask is defined with respect to its center location. 


\subsubsection{High boost filtering}

High pass filtering in terms of subtracting a low pass image from the original image, that is,

High pass $=$ Original - Low pass.

However, in many cases where a high pass image is required, also want to retain some of the low frequency components to aid in the interpretation of the image. Thus, if multiply the original image by an amplification factor A before subtracting the low pass image, will get a high boost or high frequency emphasis filter. Thus,

$$
\begin{aligned}
\text { High boost } & =\text { A. Original }- \text { Low pass } \\
& =(\text { A-1 }) \cdot(\text { Original })+\text { Original }- \text { Low pass } \\
& =(\text { A-1 }) \cdot(\text { Original })+\text { High pass }
\end{aligned}
$$

Now, if $\mathrm{A}=1$ we have a simple high pass filter. When $\mathrm{A}>1$ part of the original image is retained in the output. A simple filter for high boost filtering is given by

\begin{tabular}{|l|l|l|}
\hline$-1 / 9$ & $-1 / 9$ & $-1 / 9$ \\
\hline$-1 / 9$ & $\omega / 9$ & $-1 / 9$ \\
\hline$-1 / 9$ & $-1 / 9$ & $-1 / 9$ \\
\hline
\end{tabular}

Fig-2: High boost filtering

Where $\omega=9 \mathrm{~A}-1$.

\subsubsection{Unsharp masking}

The unsharp filter is a simple sharpening operator which derives its name from the fact that it enhances edges (and other high frequency components in an image) via a procedure which subtracts an unsharp, or smoothed, version of an image from the original image. The unsharp filtering technique is commonly used in the photographic and printing industries for crispening edges. The above two techniques resultant images are having their back ground intensity levels are near to black, but sometimes we require sharpness enhancement in the image itself, for this case unsharp masking algorithm is use full.

The unsharp masking algorithm can be described by the equation: $v=y+y(x-y)$ where $\square$ is the input image, $\square$ is the result of a linear low-pass filter, and the gain $(\square>0)$ is a real scaling factor. The detail signal $\square=(\square-\square)$ is usually amplified to increase the sharpness. However, the signal contains 1) details of the image, 2) noise, and 3) overshoots and under-shoots in areas of sharp edges due to the smoothing of edges. While the enhancement of noise is clearly undesirable, the enhancement of the under-shoot and overshoot creates the visually unpleasant halo effect. Ideally, the algorithm should only enhance the details of an image. Due to this reason we require that the filter is not sensitive to noise and does not smooth sharp edges. The edge preserving filters, such as nonlinear filters, cubic filter and are used to replace the linear low-pass filter. The former is less sensitive to noise and the latter does not smooth sharp edges. To reduce the halo effect, edge-preserving filters such as: adaptive Gaussian filter, weighted least-squares based filters, non-local means filter, and bilateral filters are used. An important problem associated with the unsharp masking algorithm is that the result is usually out of the range. For example, for an 8-bit image, the range is [0,255]. A careful rescaling process is usually needed for each image.

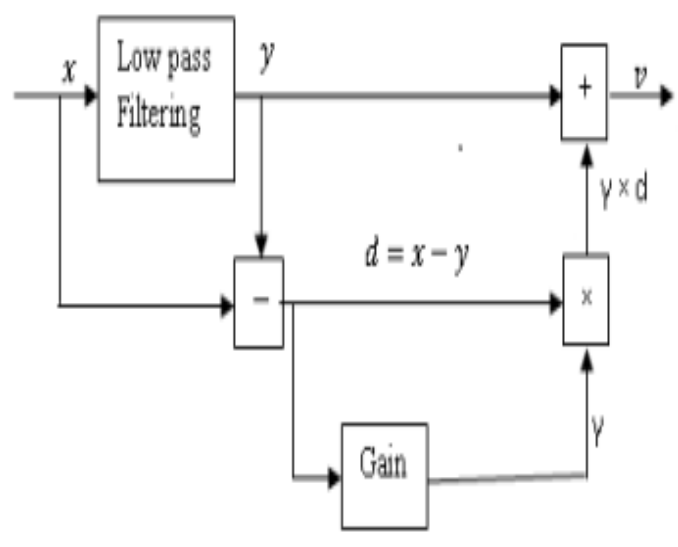

Fig-3:.Block Diagram of Classical Unsharp Masking

\subsection{Contrast Enhancement}

Contrast is a basic perceptual feature of an image .It is Difficult to see the details in a low contrast image. To improve the contrast or to enhance the contrast the adaptive histogram equalization is frequently used. To enhance the contrast recently some new advanced algorithms are developed, which is retinex based algorithms.

\subsubsection{Generalized Linear System}

Before going to develop an effective computer vision technique one must consider 1) Why the particular operations are used, 2) How the signal can be represented and, 3) What implementation structure can be used. And there is no reason to continue with usual addition and multiplication. For digital signal processing, via abstract analysis we can create more easily implemented and more generalized or abstract versions of mathematical operations. Due to the result of this creation, abstract analysis may show new ways to creating systems with desirable properties. Following these ideas, the generalized linear system, shown in Fig. 4, is developed.

The generalized addition and scalar multiplication operations denoted by $\bigoplus$ and $\Theta$ are defined as follows: 


$$
x \oplus y=\emptyset^{-1}[\varnothing x+\emptyset(y)]
$$

$$
\begin{aligned}
& \text { And } \\
& \alpha \otimes x=\emptyset^{-1}[\alpha \emptyset(x)]
\end{aligned}
$$

Where $x$ and $y$ are signal samples is usually a real scalar, and $\varnothing$ is a nonlinear function.

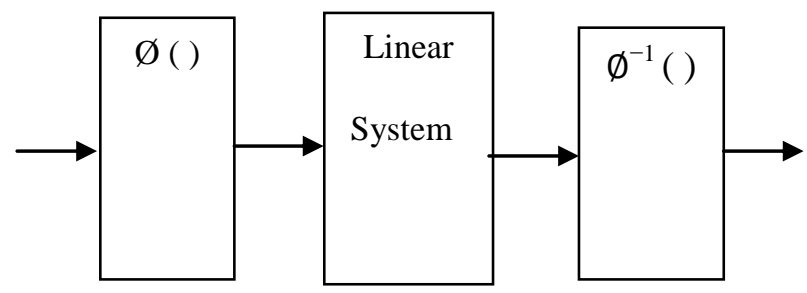

Fig-4: Block diagram of generalized linear system

The tangent approach was proposed to analytically solve the out of range problem in image restoration. The tangent approach can be understood from a generalized linear system point of view, since its operations are defined by using equations (2) and (3). A notable property of the tangent approach is that the gray scale set $\square \in(-1,1)$ is closed under the new operations.

\subsection{Motivation and Contributions}

This work is motivated by unsharp masking algorithm, an outstanding analysis of the halo effect, and the requirement of the rescaling process. In this paper the tangent operations were defined, motivated by the graceful theory of the logarithmic image processing model. The major contribution and the organization of this paper are as follows. In Section 2, we first present a frame work for the generalized unsharp masking and we described about the proposed algorithm.

We proposed a new approach to solve the out of range problem, which is tangent system it is presented in section 3 . In Section 4, we describe the details of each building block of the proposed algorithm which includes the bilateral filter, the adaptive gain control, and the adaptive histogram equalization for contrast enhancement. In Section 5, we present simulation results which are compared existing unsharp masking algorithm results. And in Section 6 Conclusion and future work are presented.

\section{FRAME WORK FOR THE PROPOSED}

\section{ALGORITHM}

\subsection{Exploratory data Model and Generalized}

\section{Unsharp Masking}

The idea behind the exploratory data analysis is to decompose a signal into two parts. One part fits a particular model, while the other part is residual. In simple way the data model is: "fit
PLUS residuals". From this definition, the output of the filtering process, denoted as $\mathrm{f}(\mathrm{x})$, can be regards as the part of the image that fits the model. Thus we can represent an image using the generalized operations as follows:

$$
x=y \oplus d
$$

Where $\mathrm{d}$ is called the detail signal ( the residual). The detail signal is defined as $d=x \ominus y$, where $\ominus$ is the generalized subtraction operation. A generalized form of the unsharp masking algorithm can be written as

$$
v=h(y) \oplus g(d)
$$

Where $\mathrm{v}$ is the output of the algorithm and both $h(y)$ and $g(d)$ could be linear or nonlinear functions. This model explicitly states that the part of the image being sharpened is the model residual. In addition, this method allows the contrast enhancement by means of a suitable processing function $h(y)$ such as adaptive histogram equalization algorithm. In this way, the generalized algorithm can enhance the overall contrast and sharpness of the image.

\subsection{The Proposed Algorithm}

The proposed algorithm, shown in Fig. 5, is based upon the classical unsharp masking algorithm. Here we address the problem of the halo effect by using an edge-preserving filter which is the bilinear filter to generate the signal $\square$. The choice of the bilinear filter is due to its relative simplicity, advanced than median filter and well studied properties such as the root signals. Other more advanced edge preserving filters such as the cubic filter and wavelet-based denoising filter and nonlocal means filter can also be used.

Here we address the problem of the need for a careful rescaling process by using new operations defined based on the tangent operations and new generalized linear system. From here the gray scale set is closed under these new operations, the out-of-range problem is clearly solved and no rescaling is needed. Here we address the new concept of contrast enhancement and sharpening by using two different processes. The image $\square$ is processed by AHE algorithm and the output is called $\mathrm{h}(\square)$. The detail image is processed by $g(d)=\gamma(d) \otimes d$ where $(\square)$ is the adaptive gain and is a function of the amplitude of the detail signal $\square$. The final output of the algorithm is then given by

$$
v=h(y) \oplus[\gamma(d) \otimes d]
$$

We can see that the proposed algorithm is a generalization of the existing unsharp masking algorithm in several ways. 


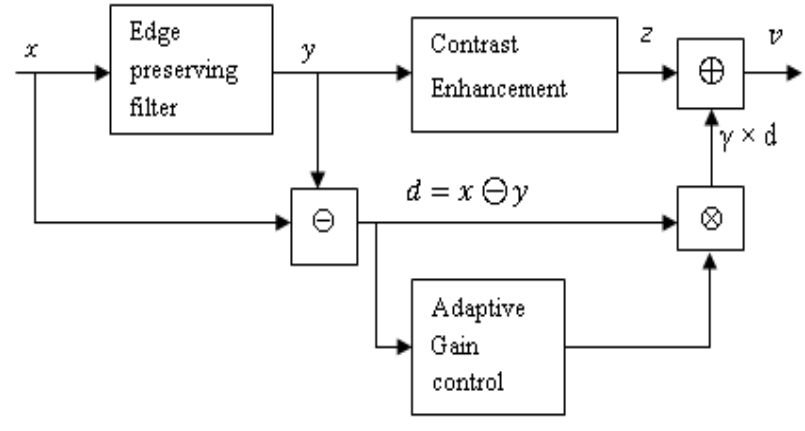

Fig-5: Block diagram of Generalized Unsharp Masking Algorithm

Here we address the problem of the need for a careful rescaling process by using new operations defined based on the tangent operations and new generalized linear system. From here the gray scale set is closed under these new operations, the out-of-range problem is clearly solved and no rescaling is needed. Here we address the new concept of contrast enhancement and sharpening by using two different processes. The image $\square$ is processed by AHE algorithm and the output is called $\mathrm{h}(\square)$. The detail image is processed by $g(d)=\gamma(d) \otimes d$ where $(\square)$ is the adaptive gain and is a function of the amplitude of the detail signal $\square$. The final output of the algorithm is then given by

$$
v=h(y) \oplus[\gamma(d) \otimes d]
$$

We can see that the proposed algorithm is a generalization of the existing unsharp masking algorithm in several ways.

\section{TANGENT OPERATION}

The operations are defined based on generalized linear system approach. We use equations (2) and (3) simplify the presentation.

\subsection{Definitions of Tangent Operations}

\subsubsection{Nonlinear Function}

For tangent operations we consider the pixel gray scale of an image $\in(-1,1)$. For an N-bit image, we can first linearly map the pixel value from the interval $[0,2 \mathrm{~N}]$ to a new interval $(-1,1)$. Then the image is processed by using tangent operations. The result is then mapped back to interval $[0,2 \mathrm{~N}]$ through the inverse mapping. We can easily verify that the signal set $\Im \in(-1,1)$ is closed under the tangent operations. The tangent system can be used as an alternative to the log-ratio to solve out-of-range problem.
In simulation we use a simple function $q(x)=\frac{2(x+1)}{2^{\mathrm{N}}+1}-1$ to map the image from $[0,2 \mathrm{~N}]$ to $(-1,1)$. The nonlinear function is defined as follows:

$$
\emptyset(x)=\frac{x}{\sqrt{1-x^{2}}}
$$

\subsubsection{Addition of two gray scales}

Using equation (2), the addition of two gray scales and is defined as

$$
x_{1} \oplus x_{2}=\frac{\emptyset\left(x_{1}\right)+\emptyset\left(x_{2}\right)}{\sqrt{1+\left(\emptyset\left(x_{1}\right)+\emptyset\left(x_{2}\right)\right)^{2}}}
$$

\subsubsection{Scalar Multiplication}

The multiplication of a gray scale $\mathrm{x}$ by real scalar is $\alpha$ between $(-\infty<\alpha<\infty)$ defined by using equation (3) as follows

$$
\alpha \otimes x=\frac{\alpha \phi(x)}{\sqrt{1+(\alpha \phi(x))^{2}}}
$$

\subsubsection{Subtraction of two gray scales}

The subtraction operation between two gray levels using the addition operation defined in equation (2) as follows:

$$
x_{1} \ominus x_{2}=\frac{\emptyset\left(x_{1}\right)-\emptyset\left(x_{2}\right)}{\sqrt{1+\left(\emptyset\left(x_{1}\right)-\emptyset\left(x_{2}\right)\right)^{2}}}
$$

\section{THE PROPOSED ALGORITHM}

\subsection{Implementation of the proposed algorithm for color Images}

In color image processing we use RGB color space images to processing. For this algorithm firstly we have to convert the color image from the RGB color space to the HSI or LAB color space. The chrominance components, such as the $\mathrm{H}$ and $\mathrm{S}$ components are not processed the luminance component I only processed. After the luminance component is processed, the inverse conversion is performed. An enhanced color image in its RGB color space is obtained. To avoid a possible problem of varying the white balance of the image when the RGB components are processed individually, we process luminance component I only.

\subsection{Enhancement of the Detail Signal}

\subsubsection{The Root Signal and the Detail Signal}

The bilateral filtering operation can be denoted as a function $y$ $=(x)$ which maps the input $x$ to the output $y$. Result image 
bilateral filter operation can be represented as root signal, which is $y$.

\subsubsection{Edge preserving filter}

A bilateral filter is an edge-preserving and noise reducing smoothing filter. The intensity value at each pixel in an image is replaced by a weighted average of intensity values from nearby pixels. This weight is based on a Gaussian distribution. Crucially the weights depend not only on Euclidean distance but also on the radiometric differences (differences in the range, e.g. color intensity or $\mathrm{Z}$ distance). This preserves sharp edges by systematically looping through each pixel and adjusting weights to the adjacent pixels accordingly. The basic idea underlying bilateral filtering is to do in the range of an image what traditional filters do in its domain. Two pixels can be close to one another, that is, occupy nearby spatial location, or they can be similar to one another, that is, have nearby values, possibly in a perceptually meaningful fashion.

Consider a shift-invariant low-pass domain filter applied to an image:

$y(h)=k_{d}^{-1} \int_{\infty}^{\infty} \int_{\infty}^{\infty} x(\xi) c(\xi-h) d \xi$

The bold font for $\mathrm{f}$ and $\mathrm{h}$ emphasizes the fact that both input and output images may be multi-band. In order to preserve the DC component, it must be

$$
k_{d}=\int_{\infty}^{\infty u} \int_{\infty}^{w 0} c(\xi) d \xi
$$

Range filtering is similarly defined:

$$
y(h)=k_{r}^{-1}(h) \int_{\infty}^{\infty} \int_{\infty}^{\infty} x(\xi) s(x(\xi)-x(h)) d \xi
$$

In this case, the kernel measures the photometric similarity between pixels.

The normalization constant in this case is

$$
k_{r}(h)=\int_{\infty}^{\infty} \int_{\infty}^{\infty} s(x(\xi)-x(h)) d \xi
$$

The spatial distribution of image intensities plays no role in range filtering taken by itself. Combining intensities from the entire image, however, makes little sense, since the distribution of image values far away from $h$ ought not to affect the final value at $h$. In addition, one can show that range filtering without domain filtering merely changes the color map of an image, and is therefore of little use. The appropriate solution is to combine domain and range filtering, thereby enforcing both geometric and photometric locality.
Combined filtering can be described as follows:

$$
y(h)=k^{-1} \int_{\infty}^{\infty \infty} \int_{\infty}^{\infty} x(\xi) c(\xi-h) s(x(\xi)-x(h)) d \xi
$$

With the normalization

$$
k(x)=\int_{\infty}^{\infty \infty} \int_{\infty}^{\infty w} x(\xi) c(\xi-h) s(x(\xi)-x(h)) d \xi
$$

Combined domain and range filtering will be denoted as bilateral filtering. It replaces the pixel value at $h$ with an average of similar and nearby pixel values. In smooth regions, pixel values in a small neighborhood are similar to each other, and the bilateral filter acts essentially as a standard domain filter, averaging away the small, weakly correlated differences between pixel values caused by noise. Consider now a sharp boundary between a dark and a bright region, as in figure 6(a).

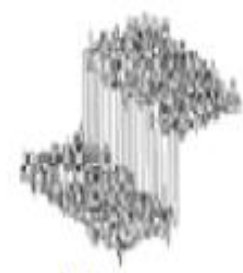

(a)

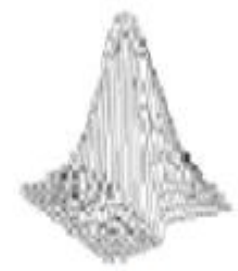

(b)

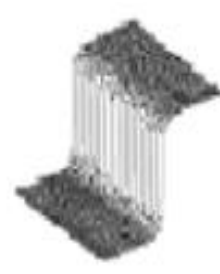

(c)
Fig-6:

When the bilateral filter is centered, say, on a pixel on the bright side of the boundary, the similarity function $\mathrm{s}$ assumes values close to one for pixels on the same side, and values close to zero for pixels on the dark side. The similarity function is shown in figure 6(b) for a $23 \times 23$ filter support centered two pixels to the right of the step in figure 6(a). The normalization term $k(h)$ ensures that the weights for all the pixels add up to one. As a result, the filter replaces the bright pixel at the center by an average of the bright pixels in its vicinity, and essentially ignores the dark pixels. Conversely, when the filter is centered on a dark pixel, the bright pixels are ignored instead. Thus, as shown in figure 6(c), good filtering behavior is achieved at the boundaries, thanks to the domain component of the filter, and crisp edges are preserved at the same time thanks to the range component.

\subsection{Contrast enhancement of the root signal}

For contrast enhancement, we use adaptive histogram equalization implemented by Matlab function in the image processing Toolbox. The function called "adapthiseq" has a parameter controlling the contrast. This parameter is determined by the user through experiments to obtain the most visually pleasing result. In our simulations, we use default values for other parameters of the function. 


\subsection{Adaptive Gain Control}

In the enhancement of the detail signal we require gain factor to yield good results, it be must be greater than one. Using a same gain for the entire image does not lead to good results, because to enhance the small details a relatively large gain is required. This large gain can lead to the saturation of the detailed signal whose values are larger than a certain threshold. Saturation is undesirable because different amplitudes of the detail signal are mapped to the same amplitude of either -1 or 1 . This leads to loss of information. Therefore, the gain must be controlled adaptively.

We describe the following below gain control algorithm using tangent operations. To control the gain, we first perform a linear mapping of the detail signal $\square$ to a new signal c,

$$
c=2 d-1
$$

Such that the dynamic range of $\square$ is $(-3,1)$. A simple idea is to set the gain as a function of the signal $\square$ and to gradually decrease the gain from its maximum value $\gamma_{M A X}$ when $|c|<T_{\text {to }}$ its minimum value $\gamma_{M I N}$ when $|c| \rightarrow 1$. More specifically, we propose the following adaptive gain control function:

$$
\gamma(c)=\alpha+\beta e^{\left(-|c|^{\eta}\right)}
$$

Where $\eta_{\text {is a parameter that controls the rate of decreasing. }}$ The two parameters $\alpha$ and $\beta$ are obtained by solving the equations: $\gamma(-1)=\gamma_{M A X}$ and $\gamma(1)=\gamma_{M I N}$. For a fixed $\eta$, we can easily determine the two parameters as follows:

$$
\gamma(c)=\left(\gamma_{M A X}-\gamma_{M I N}\right) /\left(1-e^{-1}\right)
$$

And

$$
\alpha=\gamma_{M A X}-\beta
$$

Although both $\gamma_{M A X}$ and $\gamma_{M I N}$ could be chosen based upon each individual image processing task, in general it is reasonable to set $\gamma_{M I N}=1$.

\section{RESULTS AND COMPARISON}

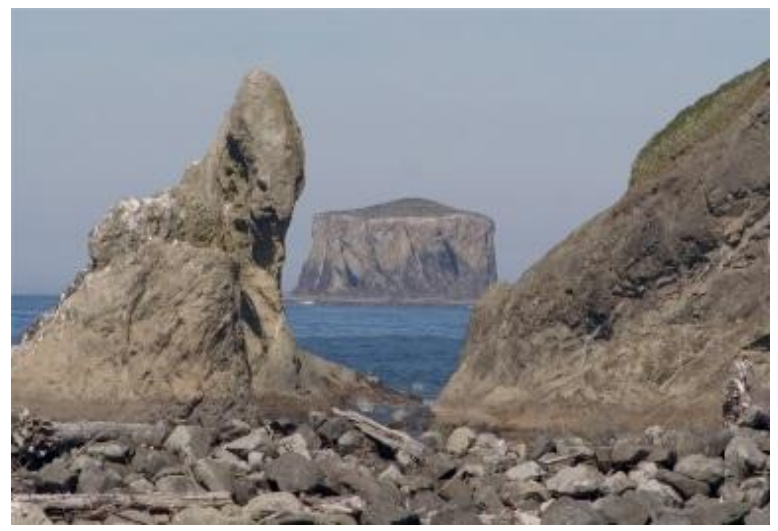

Fig-7: Original Image Rock

Figure7 shows the original image to do the simulation of this existing and proposed concepts the range of the image is $[0,255]$.

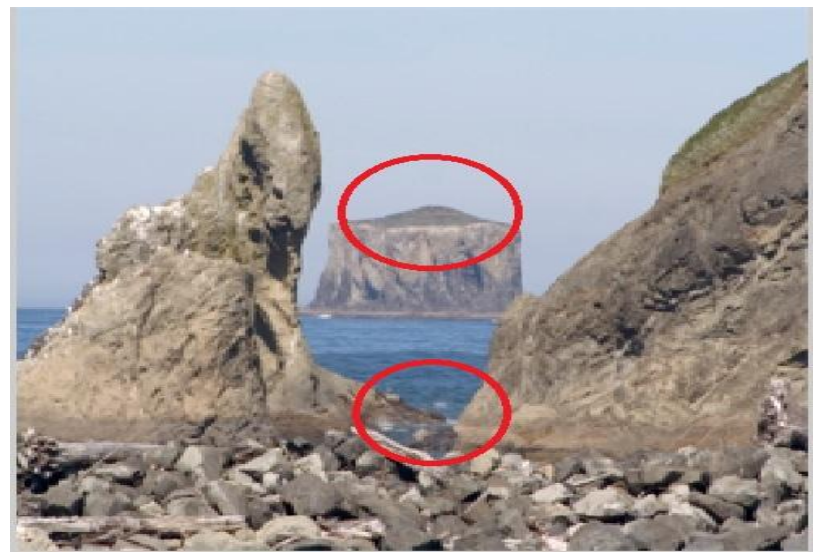

Fig-8: Result of Classical Unsharp Masking Algorithm

Figure.8. Shows the result of existing unsharp masking algorithm, it exhibit hallo effect ( the halo-effect are marked by the red ellipse) at the edges of the image, it is a drawback of existing algorithm.

Figure 9.Shows the 133th row gray level profile of resultant image of existing unsharp masking algorithm. It exhibit out of range problem. The range of selected image for processing is $[-1,1]$, but the resultant image exceeds this range this range (it suffering with out of range problem). The size of the image is 533 X 2400, means 533 rows and 2400 columns among these one of the column or row gray level profile can choose to exhibit the out of range problem., here all the pixels are not suffer without range problem, so there is no guaranty to exhibit out or range problem for all rows and columns, so choose appropriate column or row to exhibit out of range problem. 


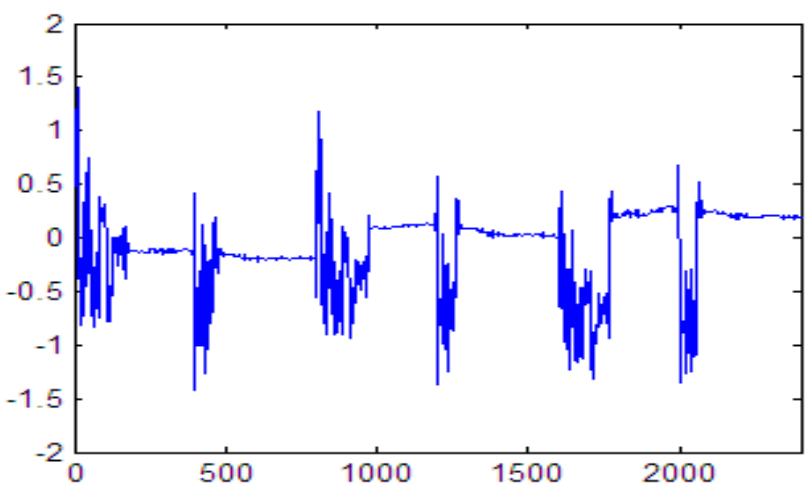

Fig-9: 133th row gray level profile of existing unsharp masking resultant image

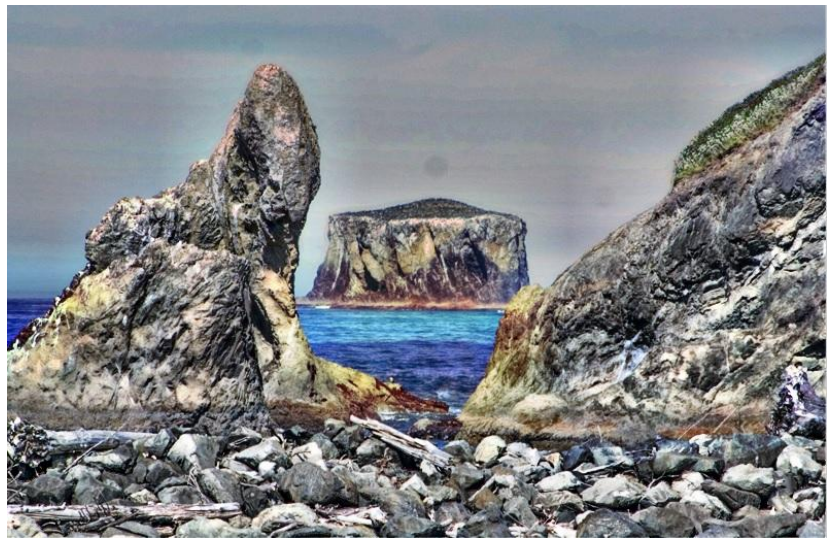

Fig-10: Result of the Proposed Algorithm

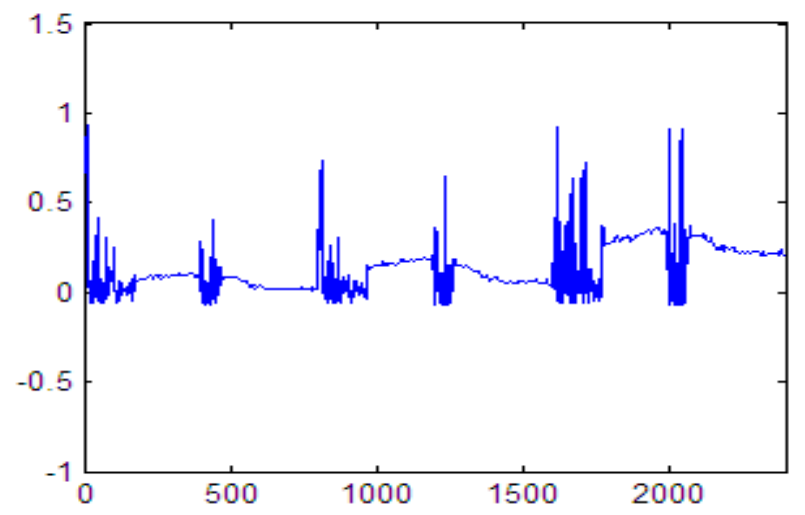

Fig-11:133th gray level profile of proposed algorithm resultant image

The result of proposed algorithm is shown in figure10. It exhibits reduced hallo-effect, enhanced low contrast details of the image, and sharpness of the image is also enhanced. The figure11.shows 133th row level profile of result of proposed algorithm, it exhibit the solved out or range problem, here the range of the image is $[-1.1]$.

\section{CONCLUSION AND FURTHER WORK}

In this paper, we developed generalized unsharp masking algorithm by using an exploratory data model as a unified frame work, it is very useful for highly texture images and which images having long distance objects to find the exact edges for that objects. By using the generalized unsharp masking algorithm we solved problems associated with existing unsharp masking algorithm, first one is the halo-effect is reduced by means of an edge-preserving filter that is bilateral filter, second one is rescaling process eliminated by using tangent operations and final one we introduced a new future that is simultaneously enhancing contrast and sharpness by means of individual treatment of the model component and the residual. Extensions of this work can be carried out in a number of directions. In this work, we only test the bilateral filter as a computationally inexpensive edge preserving filter. It is expected that other more advanced edge preserving filters such as non local means filter, the least squares filters and wavelet based denoising can produce similar or even better results.

\section{REFERENCES}

[1].G. Ramponi, - A cubic unsharp masking technique for contrast enhancement, Signal Process., pp. 211-222, 1998.

[2]. Gaung Deng, -A Generalized Unsharp Masking Algorithm, IEEE Trans on image processing, vol. 20, no. 5, May 2011.

[3]. S. J. Ko and Y. H. Lee, -Center weighted median filters and their applications to image enhancement, IEEE Trans. Circuits Syst., vol. 38, no. 9, pp. 984-993, Sep. 1991.

[4]. M. Fischer, J. L. Paredes, and G. R. Arce, -Weighted median image sharpeners for the world wide web, IEEE Trans. Image Process., vol. 11, no. 7, pp. 717-727, Jul. 2002. [5]. R. Lukac, B. Smolka, and K. N. Plataniotis, - Sharpening vector median filters, Signal Process., vol. 87, pp. 2085-2099, 2007.

[6]. A. Polesel, G. Ramponi, and V. Mathews, -Image enhancement via adaptive unsharp masking, IEEE Trans. Image Process., vol. 9, no. 3, pp. 505-510, Mar. 2000.

[7]. E. Peli, -Contrast in complex images,J. Opt. Soc. Amer.,vol. 7, no. 10, p. 2032-2040, 1990.

[8]. S. Pizer, E. Amburn, J. Austin, R. Cromartie, A. Geselowitz, T. Greer, B. Romeny, J. Zimmerman, and K. Zuiderveld, -Adaptive histogram equalization and its variations, Comput. Vis. Graph. Image Process., vol. 39, no. 3, pp. 355-368, Sep. 1987.

[9]. G. Deng and L. W. Cahill, -Image enhancement using the log-ratio approach, in Proc. 28th Asilomar Conf. Signals, Syst. Comput.s, 1994, vol. 1, pp. 198-202.

[11]. L. W. Cahill and G. Deng, -An overview of logarithmbased image processing techniques for biomedical applications, in Proc. 13th Int.Conf. Digital Signal Process. 1997, vol. 1, pp. 93-96.

[12]. J. Pinoli, -A general comparative study of the multiplicative homomorphic log-ratio and logarithmic image 
processing approaches, Signal Process., vol. 58, no. 1, pp. 1145, 1997.

[13]. M. Jourlin and J.-C. Pinoli, -A model for logarithmic image processing, J. Microsc., vol. 149, pp. 21-35, 1988.

[14]. Digital Image Processing-R.C.Gonzalez\&Woods, Addition Wesley/Pearson education, 2nd Edition, 2002.

[15]. Fundamentals of Digital Image Processing-A.K.Jain, PHI.

[16]. C. Tomasi and R. Manduchi, "Bilateral Filtering for Gray and Color Images", Proceedings of the 1998 IEEE International Conference on Computer Vision, Bombay, India

\section{BIOGRAPHIES:}

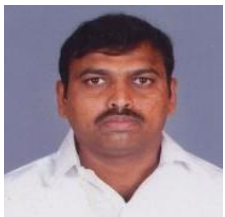

SUNKARI SRIDHAR, Assistant Professor, ECE Dept., RRSCET, Muthangi, Patancheru, Medak Dist. He received B.Tech degree from JNTU, Hyderabad and M.Tech degree from JNTUCEH, Kukatpally, Hyderabad.

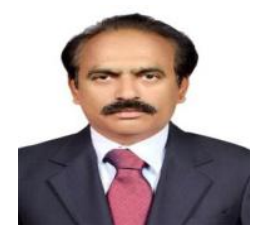

SHAIK MEERAVALI, Professor and Head, Department of Electronics and Communication Engg, RRS College of Engineering and Technology, Muthangi, Andhra Pradesh, India. 